\title{
MULTI-VIEW VIDEO COMPRESSION USING DYNAMIC BACKGROUND FRAME AND 3D MOTION ESTIMATION
}

\author{
Manoranjan Paul, Junbin Gao, Michael Anotolovich, and Terry Bossomaier \\ School of Computing and Mathematics, Charles Sturt University, Bathurst, Australia \\ Email: \{mpaul, jbgao, mantolovich, tbossomaier\}@,csu.edu.au
}

\begin{abstract}
The H.264/MVC multi-view video coding standard provides a better compression rate compared to the simulcast coding technique (i.e., H.264/AVC) by exploiting inter- and intraview redundancy. However, this technique imposes random access frame delay as well as requires huge computational time. In this paper three novel techniques are proposed to overcome the above mentioned problems. Firstly, a simulcast video coding technique is proposed where each view is encoded individually using two reference framesimmediate previous frame and a dynamic background frame (popularly known as McFIS- the most common frame in a scene) of the corresponding view. Secondly a novel technique is proposed using 3D motion estimation (3D-ME) where a $3 \mathrm{D}$ frame is formed using the same temporal frames of all views and ME is carried out for the current 3D frame using the immediate previous $3 \mathrm{D}$ frame as a reference frame. Thereafter, a fractional ME refinement is also conducted on individual frames of 3D current frame using individual reference frames. Finally, a modification of the 3D-ME technique is proposed where an extra reference frame namely 3D McFIS is used for 3D-ME. As the correlation among the intra-view images is higher compared to the correlation among the inter-view images, the proposed 3D-ME techniques reduce the overall computational time and eliminate the frame delay with comparable rate-distortion (RD) performance compared to H.264/MVC. Experimental results reveal that the proposed techniques outperform the H.264/MVC in terms of improved RD performance by reducing computational time and by eliminating the random access frame delay.
\end{abstract}

Index Terms- McFIS, 3D Motion Estimation, 3D Video Coding, uncovered background, hierarchical B-picture, and MRFs.

\section{INTRODUCTION}

In multi-view video coding (MVC), a scene is captured by different video cameras from different angles that provide a more realistic experience about the scene compared to that of a single video camera. Obviously, transmission and storing of multi-view videos require huge amounts of computation and data manipulation compared to single view video, although there is a significant amount of data redundancy among views. Recently, H.264/MVC [1]-[3] proposed a reference structure among views $(S)$ and temporal $(T)$ images. In the reference structure of MVC, hierarchical B-picture prediction format [4][5] is used for intra- and inter-view. The technique exploits the redundancy from neighbouring frames as reference from both inter- and intra-view to encode the current frame. The inter- and intraview referencing technique provides $20 \%$ more bitstream reduction compared to the simulcast technique where nointer-view redundancy is exploited i.e., each view is encoded separately [1].

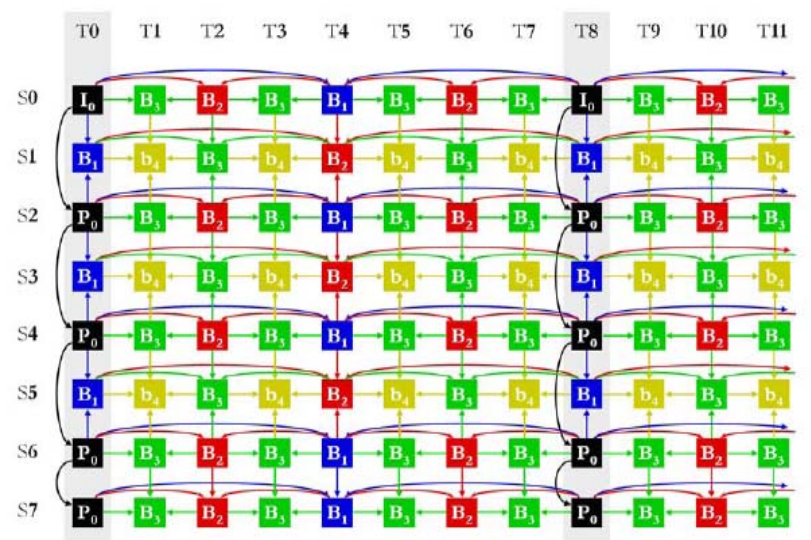

Fig 1: Prediction structure recommended by the H.264/MVC for referencing different views $(S)$ and different temporal $(T)$ images for coding.

Fig 1 shows the prediction structure of the MVC recommended by the H.264/MVC standard where eight views are used. According to the prediction structure, a frame may use maximum four frames as reference frames. Sometimes, encoding (or decoding) a frame using multiple inter- and intra-view reference frames requires encoding (or decoding) a number of frames in advanced; thus, the structure introduces random access delay due to the dependency on other frames. The random access delay is measured based on the maximum number of frames that must be decoded in order to access a B-frame in the hierarchical structure. The access delay for the highest hierarchical order is given by:

$$
F_{\text {max }}=3 \times \text { level }_{\text {max }}+2 \times\lfloor(N-1) / 2\rfloor
$$


where level $_{\max }$ is the highest hierarchical order and $N$ is the total number of views [3]. For instance, in order to access a B-frame in the 4th hierarchical order (B4-frames in Fig 1), 18 frames $\left(F_{\max }=18\right)$ must be decoded. Due to the random access problem, some applications such as interactive realtime communication may not be possible using the existing prediction structure.

The H.264/AVC video coding standard improves coding performance by reducing up to $50 \%$ bitstreams compared to its predecessor H.263 by increasing computational complexity by up to 10 times [7] for a single view video. In addition, when H.264/MVC encodes multiview videos, it requires multiple amounts of computational time compared to the H.264/AVC. The enormous requirement of computational time limits the scope of $3 \mathrm{D}$ video coding applications especially for electronic devices with limited processing and battery power such as smart phones.

Although the simulcast coding technique using H.264/AVC (where each view is encoded individually) for multi-view videos is inferior compared to H.264/MVC in terms of rate-distortion (RD) performance, it does not have the random access delay problem. Recently, a video coding technique was proposed where a dynamic background frame (i.e., McFIS- the most common frame in a scene) is used as an extra reference frame [8][9] for encoding the current frame assuming that the motion part of the current frame would be referenced using the immediate previous frame and the static background part would be referenced using McFIS. McFIS is generated using the Gaussian mixture model [10]- [12]. In this paper a simulcast video coding approach (named H.264/AVC-McFIS) based on H.264/AVC is proposed where McFIS of each view is used to encode the corresponding view. This technique improves RD performance compared to H.264/MVC and simulcast H.264/AVC on multi-view videos which have significant amounts of background area. Computational time requirement of the proposed technique should be higher compared to H.264/MVC and H.264/AVC.

In this paper a new technique is proposed using $3 D$ motion estimation (3D-ME) to overcome the random access frame delay and computational time problems of the existing MVC technique. In the 3D-ME technique, a 3D frame is formed using the same temporal frames (i.e, $i^{\text {th }}$ frames) of all views and motion estimation (ME) is carried out for a macroblock of the current 3D frame using the immediate previous 3D frame as reference frame (which is formed by the $(i-1)^{\text {th }}$ frames of all views). Thereafter, a fractional ME refinement is also conducted on individual frames of the 3D current frame using individual reference frames to capture different motions of each view. As the correlation among the intra-view images is higher than the correlation among the inter-view images, the proposed 3DME technique does not degrade the rate-distortion performance significantly, but reduces the overall computational time and eliminates the random access frame delay compared to H.264/MVC which enables interactive real time communications.

Another technique (named 3D-ME-McFIS) is also proposed in this paper where an extra reference 3D matrix comprising McFISes of all views is used for 3D-ME. Experimental results reveal that the proposed 3D-MEMcFIS technique outperforms the H.264/MVC by improving rate-distortion performance and reducing computational time without frame delay for most of video sequences.

The rest of the paper is organized as follows. Section 2 describes the proposed simulcast H.264/AVC-McFIS coding technique with details of McFIS generation steps. Section 3 explains the proposed 3D-ME video coding techniques with experimental rationalities. Section 4 describes the third proposed 3D-ME-McFIS coding techniques. Section 5 analyses computational complexity of the proposed techniques against the state-of-the-art method. Section 6 describes experimental set up and analyses experimental results, while Section 7 concludes the paper.

\section{PROPOSED SIMULCAST VIDEO CODING WITH MCFIS (H.264/AVC-MCFIS)}

The simulcast coding technique using H.264/AVC is inferior to H.264/MVC in terms of rate-distortion performance; however, the simulcast technique does not have the random access frame delay problem. Moreover, the McFIS-based coding technique outperforms the H.264/AVC technique by exploiting static and uncovered background areas. Thus, a new simulcast technique is proposed where the current frame is encoded using two reference frames immediate previous frame and a McFIS. Note that a McFIS of a view is generated using the frames of the corresponding view. The ultimate reference frame is selected at block and sub-block levels using the Lagrangian multiplier [7]. The McFIS-based coding technique outperforms H.264/AVC by mainly exploiting uncovered background areas of a scene.

Generally we consider a pixel as a part of the background if it keeps its intensity for a number of frames. Based on this assumption, dynamic background modeling (DBM) [10]-[12] is formulated. We assume that the $k^{\mathrm{th}}$ Gaussian at time $t$ representing a pixel intensity with mean $\mu_{k}^{t}$, standard deviation (STD) $\sigma_{k}^{t}$, recent value $\gamma_{k}^{t}$, and weight $\omega_{k}^{t}$ such that $\sum \omega_{k}^{t}=1$. The learning parameter $\alpha$ is used to balance the current and past values of parameters such as weight, STD, mean, etc. After initialization, for every new observation $X^{t}$ (pixel intensity at time $t$ ) is first matched against the existing models in order to find one (e.g., $k^{\text {th }}$ model) such that $\left|X^{t}-\mu_{k}^{t-1}\right| \leq 2.5 \sigma_{k}^{t-1}$. If such a model exists, then update the corresponding recent value parameter $\gamma_{k}^{t}$ with $X^{t}$. Other parameters are updated with the learning rate as: 
$\mu_{k}^{t}=(1-\alpha) \mu_{k}^{t-1}+\alpha X^{t}$

$\sigma_{k}^{t^{2}}=(1-\alpha) \sigma_{k}^{t-1^{2}}+\alpha\left(X^{t}-\mu_{k}^{t}\right)^{T}\left(X^{t}-\mu_{k}^{t}\right)$;

$\omega_{k}^{t}=(1-\alpha) \omega_{k}^{t-1}+\alpha$; and the weights of the remaining Gaussians (i.e., $l$ where $l \neq k$ ) are updated as $\omega_{l}^{t}=(1-\alpha) \omega_{l}^{t-1}$. After each iteration, the weights are normalized. If the model does not exist, a new Gaussian model is introduced with $\gamma=\mu=X^{t}, \sigma=30$, and $\omega=0.001$ by evicting the $K^{\text {th }}$ (based on $w / \sigma$ in descending order) model if it exists. For more details in modeling and model updating, please refer to [8]-[12]. To get the background pixel intensity from the above mentioned models for a particular pixel, we take the average of the mean pixel intensity and recent pixel value of the model that has the highest value of weight/standard deviation among the models of a pixel.

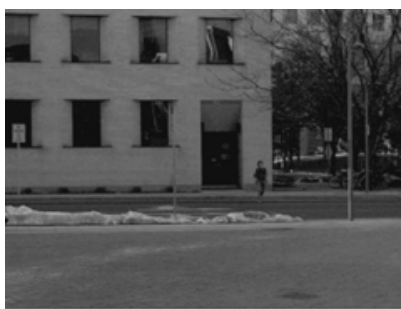

(a)

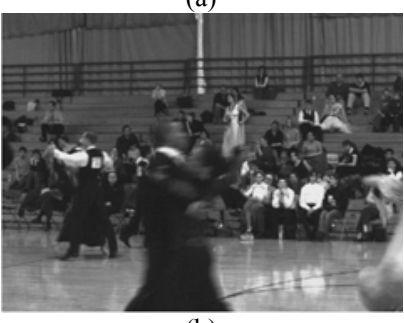

(b)

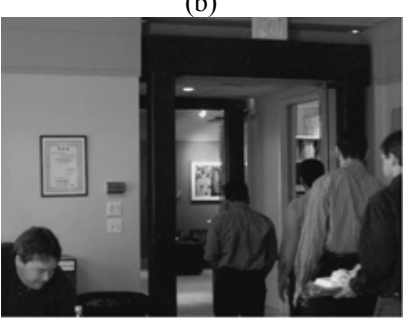

(c)

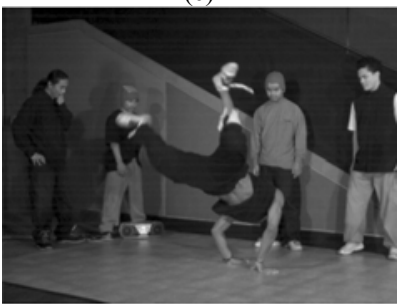

(d)

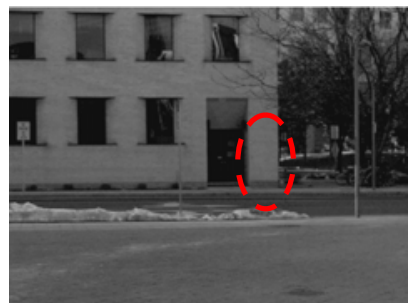

(e)

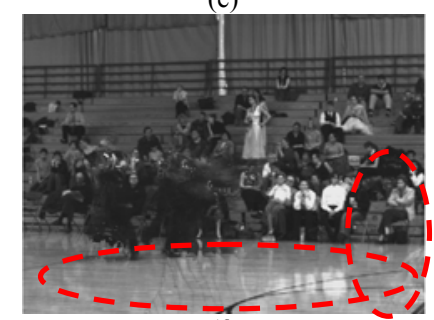

(f)

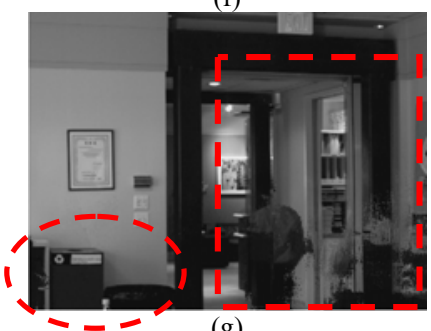

(g)

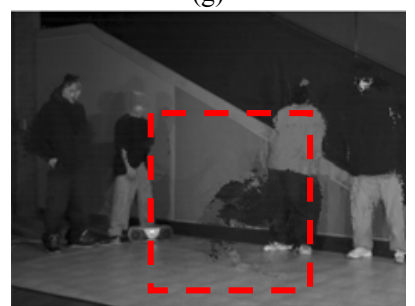

(h)
Fig 2: Examples of McFISes and uncovered/previously occluded background using Vassar, Ball Room, Exit, and Break Dancing video sequences, (a), (b), (c), and (d) an original frame of Vassar, Ball Room, Exit, and Break Dancing sequences respectively; (e), (f), (g), and (h) corresponding McFISes of the videos respectively.
Four examples of McFIS are shown in Fig 2 using frames of Vassar, Ball Room, Exit, and Break Dancing video sequences respectively. Fig 2 (a), (b), (c), \& (d) show the original frames of corresponding videos and (e), (f), (g), $\&(\mathrm{~h})$ show McFISes. The red-dotted ovals/rectangles in (e), (f), (g), \& (h) indicate the uncovered/occluded background captured by the corresponding McFIS. To capture the uncovered background by any single frame is impossible unless this uncovered background is visible for one frame and that frame is used as a second reference frame. The experimental results of computational time requirements and $\mathrm{RD}$ performance are analyzed in Section 5 and Section 6 together with other schemes. The simulcast H.264/AVCMcFIS requires more encoding time compared to H.264/MVC, however, it can outperforms the MVC technique if a video has significant amounts of background area.

\section{PROPOSED 3D MOTION ESTIMATION (3D ME) TECHNIQUE}

A scene is captured by a number of cameras which are placed at different angles in multi-view system. As the same scene is captured by all cameras, there are inter- and intraview redundancies. In general, we can assume that relative object movement within a view is very similar to other views. To find the motion similarity, we have investigated the motion vector relationship among the views of the multiview video sequences using four standard video sequences such as Exit, Ball Room, Vassar, and Break Dancing. First we determine the motion vectors of all macroblocks for each frame of a view using a $16 \times 16$ block size full search ME technique of \pm 15 search length. Then find the similarity of the motion vectors of a view with other views.

Fig 3 (a) shows average similarity of motion vectors among different views where the first 10 frames are used for each view of each sequence. The figure confirms that the similarity is $51 \%$ to $93 \%$. The experimental data indicate that the motion vector of the macroblock at the $i^{\text {th }}$ frame of the $j^{\text {th }}$ view has $51 \%$ to $93 \%$ of similarity with the co-located macroblock at the $i^{\text {th }}$ frame of other views. We have also plotted absolute motion vector differences for four standard multi-view video sequences in Fig 3 (b), (c), (d), and (e) using the first two frames. The figures confirm that a significant amount of macroblocks have zero motion vector differences between two views. To be more specific, Vassar and Ballroom sequences have more zero motion vector differences. We can exploit this relationship to avoid random access delay and computational time problems of the existing H.264/MVC prediction structure.

In the proposed 3D-ME technique, we can make a 3D frame comprising $i^{\text {th }}$ frames of all views and ME can be carried using integer search length for a 3D macroblock (another dimension is formed using co-located macroblocks from different views) where the reference 3D frame would be formed using the immediate previous i.e., $(i-1)^{\text {th }}$ frames 
of all views. Thereafter, a fractional ME refinement is also conducted on individual frames of the current 3D frame using individual reference frames to capture different motions of each view. As we have seen that for some video sequences, motion similarity is not very high, fractional ME refinement for each view has improved the $\mathrm{RD}$ performance. In the proposed 3D-ME technique, we do not exploit inter-view redundancy explicitly, due to the following three reasons: (i) the correlation among the intraview images is higher than the correlation among the interview images [1]-[3], (ii) to avoid random access frame delay, and (iii) to reduce computational time.

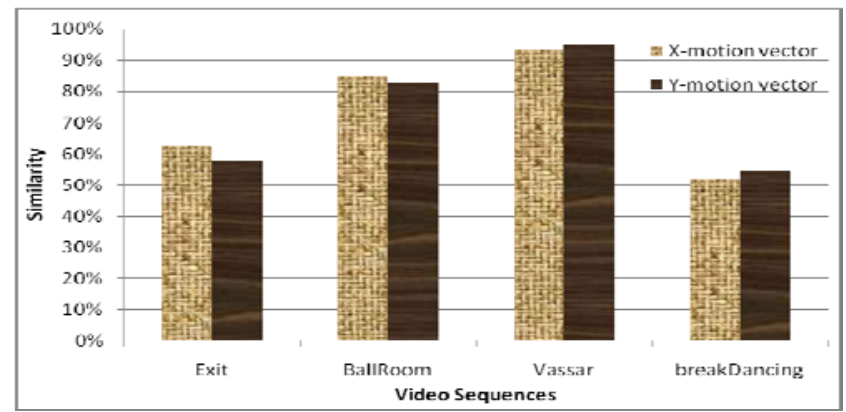

(a)

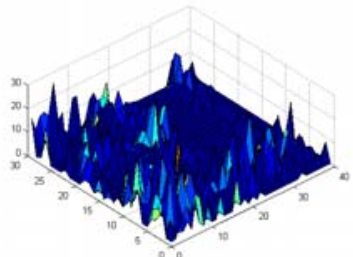

(b)

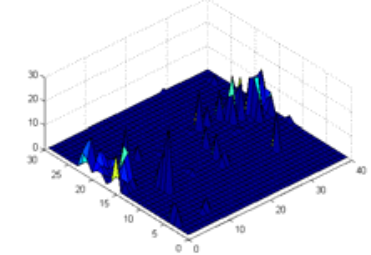

(d)

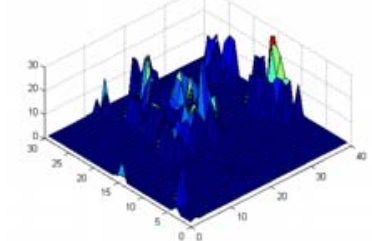

(c)

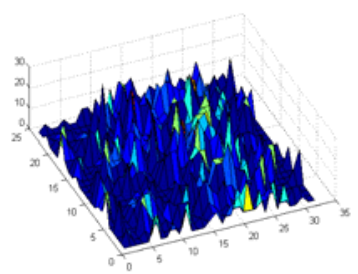

(e)
Fig 3: Motion relationship among multi-view frames: (a) average similarity of the motion vectors among different views for four standard multi-view video sequences where the first 10 frames are used for each view of each sequence; (b) (c) (d) and (e) motion vector differences among two views for Exit, Ballroom, Vassar, and Break Dancing video sequences respectively.

Fig 4 shows the formation of the 3D frame using $i^{\text {th }}$ frames and $(i-1)^{\text {th }}$ frames of all views where the first $3 \mathrm{D}$ frame is the current $3 \mathrm{D}$ frame and the later is the reference $3 \mathrm{D}$ frame (where the third dimension is formed using $i^{\text {th }}$ and $(i-1)^{\text {th }}$ frames from different views respectively). We will discuss the other 3D reference frame in Section 4.

The proposed method (3D-ME) does not require any disparity estimation [13] for inter-views as we do not explicitly use any inter-view relationships. Instead of multiple motion estimation for each reference frame (e.g., B4-frame of S3 view at T3 position in Fig 1 requires 4 times the motion estimation using 4 reference frames), the proposed method requires only one motion estimation. A significant reduction in computational time can be achieved using the proposed method as it does not need disparity estimation and motion estimation for multiple reference frames. The proposed method does not require any frame delay for random access which is another benefit of it compared to the existing prediction structure as all frames at $T_{i}$ are available for encoding/decoding $T_{i+l}$ frames (see Fig $1)$.

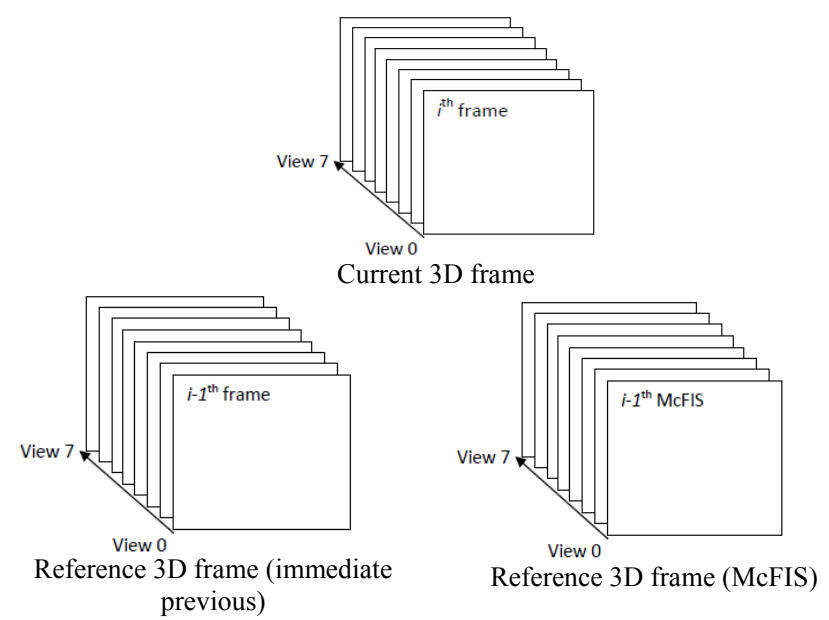

Fig 4: Different 3D frames for motion estimation and compensation for the proposed (3D-ME-McFIS) method where ME\&MC of a macroblock in a current 3D frame (comprises $i^{\text {th }}$ frames of all views) is carried out using both reference 3D frame (comprises $(i-1)^{\text {th }}$ frames of all views) and 3D McFIS (comprises all McFISes of views up to $(i-1)^{\text {th }}$ frames).

\section{PROPOSED 3D MOTION ESTIMATION TECHNIQUE WITH 3D MCFIS (3D-ME-MCFIS)}

Although the proposed 3D-ME method successfully overcomes two limitations such as computational time and random access frame delay, it (with its current state) could not outperform H.264/MVC in terms of rate-distortion performance as the experimental results (see Fig 3) reveal that the motion vector similarity is not $100 \%$. The experimental results also reveal that some cases such as motion active video sequences (exit and Break Dancing), the motion vector similarity is around $50 \%$. In results, the proposed method degrades the rate-distortion performance for those cases. It is also worthy to investigate the utilization of the computational gain of the proposed method for improving the rate-distortion performance without sacrificing computational gain and random access delay.

As mentioned earlier, McFIS can successfully capture a static background including occluded background areas (if expressed once) from a scene of a video sequence. We have formed 3D McFIS using the McFISes of all views and then 
used it as a second reference frame when 3D motion estimation was carried out for the current 3D frame. The proposed 3D-ME-McFIS technique uses additional reference frames compared to the proposed 3D-ME technique. Obviously the 3D-ME-McFIS technique requires additional computational time compared to the 3D-ME technique due to the McFIS modelling and extra motion estimation using 3D McFIS, however, better rate-distortion performance is achieved due to foreground (using the immediate 3D previous frame) and background (using 3D McFIS) referencing.

In the 3D-ME-McFIS technique, after encoding a 3D frame, we have updated 3D McFIS by updating individual McFIS (for each view) using the latest encoding frame of the corresponding views. For example $(i-1)^{\text {th }} 3 \mathrm{D}$ McFIS is used while $i^{\text {th }} 3 \mathrm{D}$ current frame is encoded and the $i^{\text {th }} 3 \mathrm{D}$ McFIS is updated using the $i$ th encoded frames. The benefit of the updated 3D McFIS is to keep the McFIS relevant in terms of referencing.

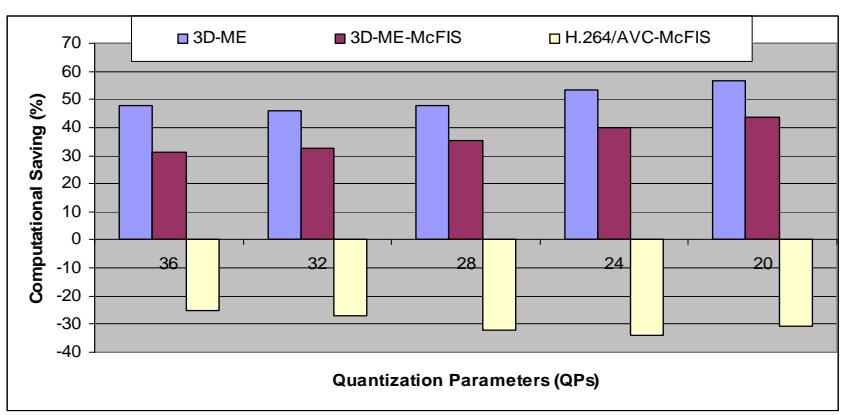

(a)

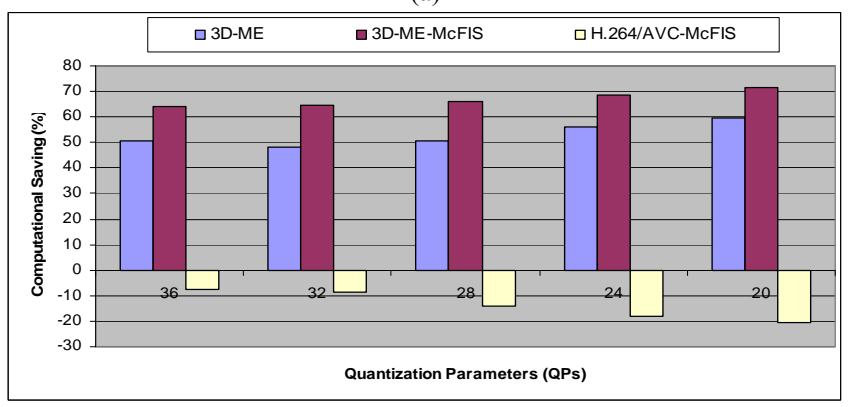

(b)

Fig 5: Average computational complexity reduction by the proposed methods (3D-ME, 3D-ME-McFIS, simulcast H.264/AVC-McFIS) against H.264/MVC using four video sequences where search length (a) 15 and (b) 31 are used.

\section{COMPUTATIONAL COMPLEXITY}

One of the objectives of the proposed methods is to reduce the computational time of the existing multi-view video coding standard to enhance the scope of 3D video coding applications. Fig 5 shows computational time comparisons among the proposed methods and the existing video coding standard using two search lengths (i.e., 15 and 31) on four standard video sequences. The figure reveals that the proposed methods (3D-ME, 3D-ME-McFIS, and H.264/AVC-McFIS) reduced around 51\%, 37\%, and $-30 \%$ of the computational time compared to H.264/MVC standard when a search length 15 was used. The corresponding figure is $53 \%, 67 \%,-13 \%$ while a search length 31 was used. Experiments were conducted on a PC with Intel(R) Core (TM) 2 CPU 6600@2.40 GHz, 2.39 $\mathrm{GHz}$, and $3.50 \mathrm{~GB}$ of RAM. Note that the proposed H.264/AVC-McFIS required $30 \%$ and $13 \%$ more computational time compared to H.264/MVC for 15 and 31 search length respectively. The proposed 3D-ME-McFIS is better compared to other schemes in terms of computational time while larger search length used as the McFIS modelling requires fixed amount of operations and it does not depend on search length. We also note that the first three views were used to calculate the results. Due to the fixed amount of operations requirement for McFIS modelling, the proposed 3D-ME-McFIS method reduced computational time slightly less compared to that of the proposed 3D-ME scheme. When large search length was used, the computational time requirement for the McFIS modelling was negligible compared to the motion estimation. The proposed 3D-ME-McFIS scheme used a small search length (e.g., 2) for the motion estimation using the McFIS as the McFIS was only used for referencing the background which had no motion. Thus, the computational time reduction for both methods is almost the same for large search length.

\section{EXPERIMENTAL RESULTS}

To compare the performance of the proposed schemes (H.264/AVC-McFIS, 3D-ME and 3D-ME-McFIS), we have implemented all the algorithms based on the H.264/MVC recommendations with $25 \mathrm{~Hz}, \pm 15$ as the search length with quarter-pel accuracy and with 16 as the GOP size. In the proposed 3D-ME scheme, we have considered the IBBP prediction format, in the proposed 3D-ME-McFIS method, we have only used the IPPP format, whereas we have used the hierarchical B-picture predication structure for H264/MVC and H.264/AVC-McFIS schemes. Obviously the proposed H.264/AVC-McFIS and 3D-ME-McFIS techniques will take some extra operations to generate McFIS. We used the same technique for modeling McFIS at the encoder and decoder, thus, we do not need to encode and transmit the McFISes to the decoder.

Fig 6 shows rate-distortion performance using H.264/MVC and the three proposed schemes the H.264/AVC-McFIS, 3D-ME and 3D-ME-McFIS using first three views of four standard multi-view video sequences. The figure reveals that the rate-distortion performance of the proposed 3D-ME scheme is comparable to H.264/MVC. However, the proposed 3D-ME scheme outperforms H.264/MVC by reducing computational time by $37 \sim 67 \%$ (see Fig 5) and eliminating random access delay. The proposed 3D-ME-McFIS scheme outperforms H.264/MVC in terms of rate-distortion performance (Break Dancing is an exception) by improving PSNR by more than $0.25 \mathrm{~dB}$, 
computational complexity by reducing the time by more than $51 \%$, and eliminating random access frame delay. Due to the huge motions (background modeling is less effective), the proposed 3D-ME-McFIS method does not outperform H.264/MVC for the Break Dancing sequence. The proposed H.264/AVC-McFIS scheme outperforms the H.264/MVC in terms of rate-distortion performance; however, it takes more computational time i.e., from $13 \%$ to $30 \%$ compared to H.264/MVC.
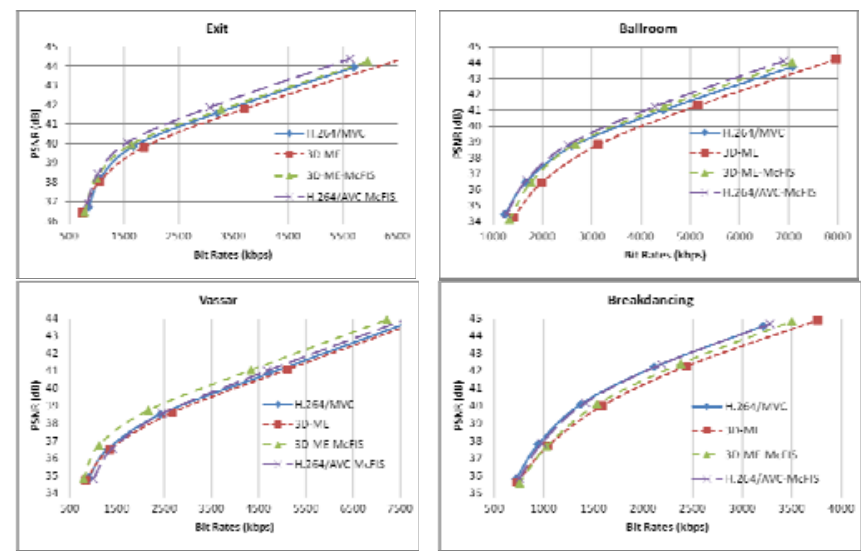

Fig 6: Rate-distortion performance by H.264/MVC and the proposed schemes (3D-ME, 3D-ME-McFIS, and H.263/AVC-McFIS) using four standard video sequences namely Exit, Ball Room, Vassar, and Break Dancing.

\section{CONCLUSIONS}

In this paper, we proposed a new 3-Dimensional motion estimation and motion compensation scheme to reduce the computational time and eliminate the random access frame delay of the existing H.264/MVC multi-view video coding standard. To eliminate random access frame delay, firstly we have proposed a simulcast McFIS-based technique on the H.264/AVC platform. The proposed technique outperforms H.264/MVC in terms of RD performance; however, it takes $30 \%$ more computational time. In the proposed 3D-ME technique, a 3D frame is formed using the same temporal frames of all views and motion estimation is carried out for a block of the current 3D frame using the immediate previous 3D frame as reference frame. A motion estimation refinement for individual current images is also conducted after integer level 3D motion estimation to improve the RD performance. This technique outperforms the existing standard by reducing computational time by more than $51 \%$ and eliminating random access frame delay without degrading the rate-distortion performance significantly compared to the state-of-the-art method i.e., H.264/MVC. This paper also proposes another technique (3D-ME-McFIS) where an extra 3D reference frame is used in addition to the immediate previous $3 \mathrm{D}$ frame. The extra $3 \mathrm{D}$ frame is formed using dynamic background frames of each view which are popularly known as McFISes (the most common frame in a scene) based on Gaussian mixture modelling. The experimental results reveal that 3D-MEMcFIS outperforms the H.264/MVC coding standard by improving PSNR by $0.25 \mathrm{~dB}$, by reducing computational time by $51 \%$, and by eliminating random access frame delay compared to the existing H.264/MVC multi-view video coding. The proposed techniques enhance the $3 \mathrm{D}$ video coding application scope for interactive real time video communications.

\section{REFERENCES}

[1] A. Vetro, T. Wiegand, and G. J. Sullivan, "Overview of the Stereo and Multiview Video Coding Extensions of the H.264/MPEG-4 AVC Standard," Proceedings of the IEEE, 99(4), 626 - 642, 2011.

[2] P. Pandit, A. Vetro, Y. Chen, "Joint Multiview Video Model (JMVM) 7 Reference Software," N9579, MPEG of ISO/IEC JTC1/SC29/WG11, Antalya, Jan. 2008.

[3] M. Talebpourazad, "3D-TV content generation and multiview video coding, $\mathrm{PhD}$ thesis, 2010.

[4] H. Schwarz, D. Marpe, and T. Wiegand, "Analysis of hierarchical B-pictures and MCTF," IEEE International Conference on Multimedia and Expo, pp. 1929-1932, 2006.

[5] M. Paul, W. Lin, C. T. Lau, and B. -S. Lee, "McFIS in hierarchical bipredictive picture-based video coding for referencing the stable area in a scene," IEEE International conference on Image Processing (IEEE ICIP-11), 2011.

[6] T. Wiegand, G. J. Sullivan, G. Bjøntegaard, and A. Luthra, "Overview of the H.264/AVC Video Coding Standard ," IEEE Transaction on Circuits and Systems for Video Technology, vol. 13, no. 7, pp. 560-576, 2003.

[7] M. Paul, M. Frater, and J. Arnold, "An efficient Mode Selection Prior to the Actual Encoding for H.264/AVC Encoder," IEEE Transaction on Multimedia, vol. 11, no. 4, pp. 581-588, June, 2009.

[8] M. Paul, W. Lin, C. T. Lau, and B. -S. Lee "Explore and model better I-frame for video coding," IEEE Transaction on Circuits and Systems for Video Technology, 2011.

[9] M. Paul, W. Lin, C. T. Lau, and B. -S. Lee, "Video coding using the most common frame in scene," IEEE International conference on Acoustics, Speech, and Signal processing (IEEE ICASSP-10), pp. 734-737, 2010.

[10] C. Stauffer and W. E. L. Grimson, "Adaptive background mixture models for real-time tracking," IEEE CVPR, vol. 2, 246-252, 1999.

[11] D.-S. Lee, "Effective Gaussian mixture learning for video background subtraction," IEEE Transactions on PAMI, 27(5), pp. 827-832, May 2005.

[12] M. Haque, M. Murshed, and M. Paul, "On Stable Dynamic Background Generation Technique using Gaussian Mixture Models for Robust Object Detection," IEEE International Conference on Advanced Video and Signal Based Surveillance (IEEE AVSS-08), pp. 41-48, 2008.

[13] X. Li, D. Zhao, S. Ma, and W. Gao, "Fast disparity and motion estimation based on correlations for multi-view video coding," IEEE Transactions on Consumer Electronics, 54(4), pp. 2037-2044, 2008. 